http://dx.doi.org/10.35381/r.k.v6i12.1378

\title{
Desaponificado de la Quinua en productos elaborados con una máquina escarificadora
}

BipDeponification of Quinoa in products made with a scarifying machine

\author{
Juan Carlos Shunta-Ronquillo \\ icshunta.r@sic-ec.com \\ Soluciones, Ingeniería y Construcción. SIC.SA, Quito \\ Ecuador \\ https://orcid.org/0000-0001-9088-904x
}

Eduardo Francisco García-Cabezas

egarcia@espoch.edu.ec

Escuela Superior Politécnica de Chimborazo. Grupo de Investigación \& Vinculación AUTOPRO, Riobamba

Ecuador

https://orcid.org/0000-0002-3547-472X

Jhonny Marcelo Orozco-Ramos

jhonny.orozco@espoch.edu.ec

Escuela Superior Politécnica de Chimborazo. Grupo de Investigación \& Vinculación AUTOPRO, Riobamba

Ecuador

https://orcid.org/0000-0003-2016-0426

Juan Carlos Cayán-Martínez

jcayan@espoch.edu.ec

Escuela Superior Politécnica de Chimborazo. Grupo de Investigación \& Vinculación AUTOPRO, Riobamba

Ecuador

https://orcid.org/0000-0001-9573-3706

Recepción: 15 de marzo 2021

Revisado: 15 de mayo 2021

Aprobación: 15 de junio 2021

Publicación: 01 de julio 2021 


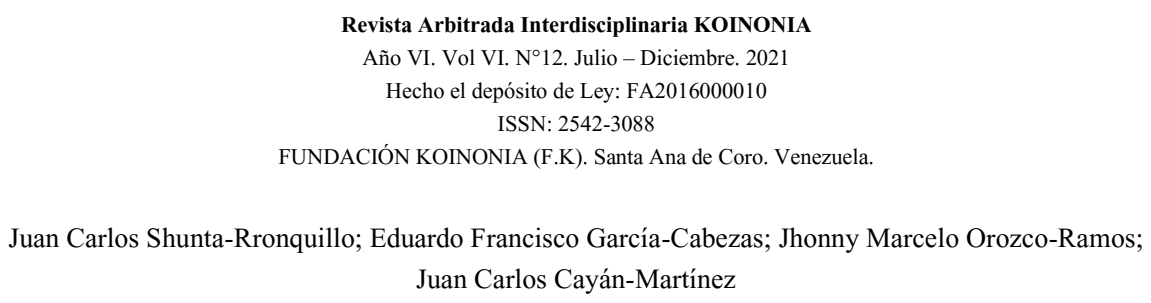

\title{
RESUMEN
}

La investigación describe la optimización del desaponificado de la Quinua para la elaboración de productos derivados mediante la implementación de una máquina escarificadora en la empresa ASOALIENU. Al respecto se procedió a establecer los materiales y elementos mecánicos basados en la norma UNE-EN 1672-2 y UNE-EN ISO 12100 vigentes en Ecuador. Se realizó el análisis estático de la estructura de soporte de maquinaria en el software SolidWorks para determinar si el diseño cumple con los parámetros técnicos de resistencia de materiales. Se efectuó la construcción de la escarificadora y realizaron pruebas de funcionamiento para determinar la capacidad de producción, además se realizó un análisis comparativo entre el método rudimentario y el método mecánico, obteniendo como resultado la reducción del tiempo de procesamiento en un $49,18 \%$, de esta manera se demuestra la mejora y optimizado del proceso reduciendo los tiempo en las operaciones de escarificado.

Descriptores: Diseño; rehabilitación; interfaz de ordenadores. (Palabras del Tesauro UNESCO).

\begin{abstract}
The research describes the optimization of the deponification of Quinoa for the elaboration of derived products through the implementation of a scarifying machine in the company ASOALIENU. In this regard, the materials and mechanical elements were established based on the UNE-EN 1672-2 and UNE-EN ISO 12100 standards in force in Ecuador. Static analysis of the machinery support structure was performed in SolidWorks software to determine if the design meets the technical parameters of strength of materials. The construction of the scarifier was carried out and operational tests were carried out to determine the production capacity, in addition, a comparative analysis was carried out between the rudimentary method and the mechanical method, obtaining as a result a reduction of processing time by $49.18 \%$, In this way, the improvement and optimization of the process is demonstrated, reducing the time in scarifying operations.
\end{abstract}

Descriptors: Design; rehabilitation; computer interface. (Words from UNESCO Thesaurus). 


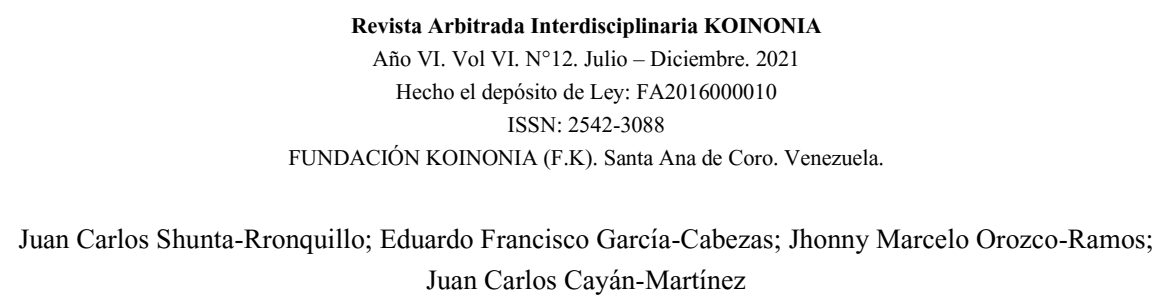

\section{INTRODUCCIÓN}

En nuestro país el Ecuador, la automatización o mecanización de los trabajos en área de la agricultura tiene un porcentaje relativamente bajo, siendo una de las limitantes y principal problema los costos de la maquinaria agrícola, las cuales solo pueden ser adquiridas básicamente por los grandes empresarios, quienes poseen el capital suficiente para su adquisición. (Alarcón Porras, 2014, p. 23). La utilización de la maquinaria agrícola puede ayudar a los pequeños y medianos productores de quinua a procesar su producto y comercializarlo directamente sin intermediarios y de esta manera obteniendo mayores ingresos, la adquisición de una tecnología para el escarificado ayuda a darle un valor agregado y mejora la calidad de los productos además optimiza la productividad de las personas quienes se dedican a la agricultura. Existen distintos tipos de máquinas escarificadoras en el mercado, que forman parte de líneas completas de procesamiento de quinua, estas máquinas procesadoras tienen un elevado costo lo cual dificulta a muchos productores del cereal la adquisición de este sistema de procesamiento. Estas líneas de procesamiento por lo general son fabricadas para las grandes industrias, esto evita que muchas asociaciones de agricultores vendan su producto directamente, obligándolos a vender el cereal a las grandes industrias.

En razón de lo anterior, se presenta esta investigación tiene como objetivo general Optimizar la desaponificado de la Quinua para elaboración de productos derivados mediante la implementación de una máquina escarificadora en la empresa ASOALIENU. En este sentido se pueda elaborar, distribuir directamente su producto en el mercado nacional e internacional. Para el diseño se elabora un diagrama de caja negra, el cual se usará para entender cómo llega el cereal a la máquina escarificadora para luego con base en estos datos adquiridos, partir al cálculo y forma de cada elemento que pertenece a la máquina, los cálculos que se desarrollados fueron la mediante la aplicación de varias asignaturas recibidas durante la formación académica, además para verificar los resultados obtenidos de algunas partes de la maquina se realiza una simulación de cargas y esfuerzos que ayuda en la 


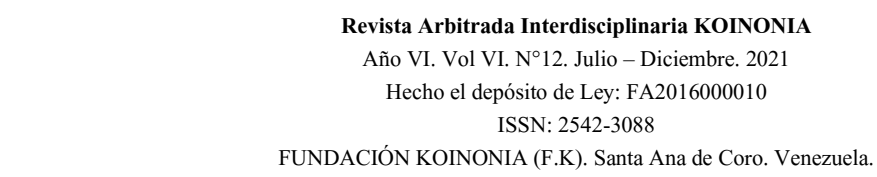

Juan Carlos Shunta-Rronquillo; Eduardo Francisco García-Cabezas; Jhonny Marcelo Orozco-Ramos;

Juan Carlos Cayán-Martínez

visualización y comprobación del elemento diseñado, para obtener una máquina que cumpla satisfactoriamente el proceso de escarificación, para el cual fue creada.

\section{Antecedentes}

Una de las principales limitantes de la producción de quinua en Ecuador es la falta de tecnología para la cosecha y procesamiento, la misma que se debe realizarse en época oportuna para evitar pérdidas de grano en el campo.

Uno de los estudios más interesantes dentro de este tema es, la escarificadora diseñada y construida por (Torres, 1980., pp. 11-16). En este caso, los granos de quinua son sometidos a un proceso combinado de efecto abrasivo y golpeado, con paletas giratorias sobre tamices estacionarios, los que recogen y separan el polvillo de saponina de los granos. La máquina consta de tres cilindros dispuestos en tres rodillos, de tal forma que los granos en proceso pasan de un cilindro a otro por gravedad. Cada cilindro está provisto de 9 paletas escarificadoras y de 12 paletas transportadoras colocadas sobre el eje giratorio. Los granos que salen del último cilindro, reciben una corriente de aire, que ayuda a separar el polvo y afrechillo, antes de ser recogidos en la salida final. Según los autores, esta máquina escarificadora tiene una eficiencia del $95 \%$ y, los contenidos de saponina en el producto final oscilan entre el 0,04 y 0,25 \%, dependiendo de la variedad utilizada como materia prima. (Candia Danz, 2016, p. 17)

En el INIAP, Ecuador, se adaptó una máquina peladora de sorgo, para la escarificación de quinua, con resultados aceptables. El principio de funcionamiento de esta máquina es la fricción del grano en un cilindro cerrado, en cuyo interior están conectadas cinco piedras de carburo de silicio, las que giran en la misma dirección y accionan un movimiento circular a los granos de quinua los que se escarifican por fricción entre las paredes del cilindro y las piedras en movimiento.

En Bolivia, el Centro Promoción de tecnologías Sostenibles, desde el año 2010, tuvo la iniciativa de construir un grupo de tres sistemas de maquinarias que se encargan de procesar la quinua en la post cosecha, cabe resaltar los sistemas de limpieza de la 


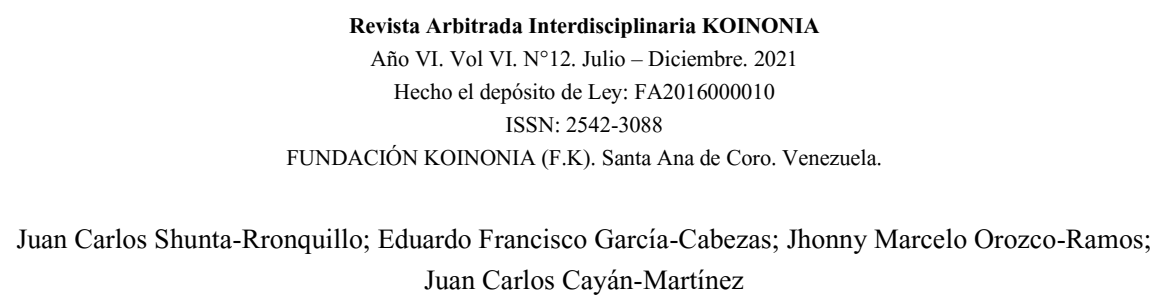

saponina por vía seca y la húmeda, que cumplen la función de eliminar en gran porcentaje el Episperma del grano y residuos sólidos de pequeñas dimensiones. El Sistema de limpieza por vía húmeda, CPTS-LVH160 (patente pendiente) cumple la función de eliminar la saponina remanente en el grano de quinua, así como toda posible piedrecilla que no haya sido eliminada por la vía seca. (Orozco, 2014)

En Salta, Argentina, en marzo 2013, Margarita Armada, Jorge A. Chavarría y Arnaldo V. Trejo diseñaron y construyeron un prototipo escarificador de quinua, este equipo contaba un su interior un tornillo escarificador, como pieza principal, mediante movimientos giratorios en el tambor y la fricción entre granos se obtuvo los resultados de $0,05 \%$ de concentración de saponina, valor que se encuentra dentro de los rangos permitidos para la venta y consumo humano. (Ramos, 2006)

\section{La Quinua}

La quinua (Chenopodium quinoa) tiene una antigüedad, por lo menos, de 5000 años como planta cultivada la quinua se cultiva en el Perú, en Bolivia, Ecuador, Chile, Argentina y Colombia. La quinua era considerada un alimento sagrado, siendo empleada, además, para usos medicinales. La quinua pertenece a la familia Chenopodiaceae. Es una planta anual de tamaño entre 1 y 2,5 metros. La panoja tiene entre 15 y $70 \mathrm{~cm}$ y puede llegar a un rendimiento de $200 \mathrm{~g}$ de granos por panoja. Las semillas pueden ser blancas, cafés, amarillas, grises, rosadas, rojas o negras y se clasifican según su tamaño en grandes $(2,2-2,6 \mathrm{~mm})$, medianas $(1,8-2,1 \mathrm{~mm})$ y pequeñas (menos de 1,8mm). (Repo de Carrasco, 2008, p. 76)

\section{Fruto y semilla}

El fruto está constituido del pericarpio (capa del fruto) y la semilla. El pericarpio está adherido a la capa de las semillas y el nivel de adherencia es variable, tiene alveolos en su superficie y la saponina que le da el sabor amargo al grano. El fruto puede alcanzar un diámetro de 1.5 a $3 \mathrm{~mm}$. Con respecto a la semilla de la Quinua está constituida principalmente por tres partes bien definidas que son: 


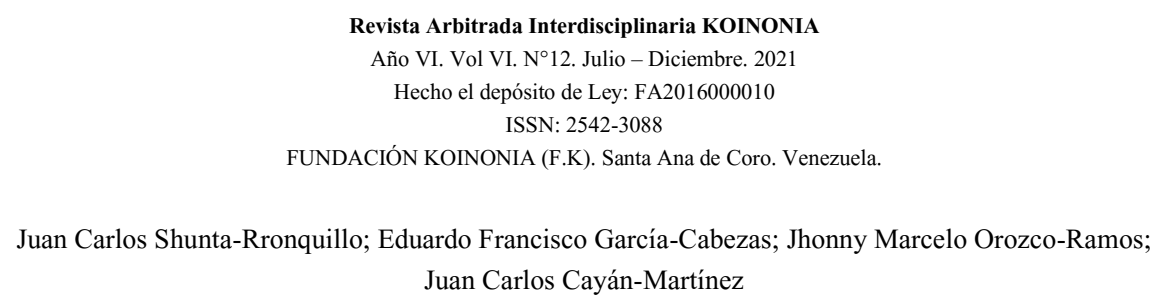

Episperma: En ella se ubica la saponina que le da el sabor amargo al grano y cuya adherencia a la semilla es variable con los genotipos (Villacorta, 1976)

Embrión: está formado por dos cotiledones y la radícula y constituye el $30 \%$ del volumen total de la semilla, el cual envuelve al perisperma como un anillo, con una curvatura de $320^{\circ}$, es de color amarillo, cuya longitud puede alcanzarlos $3,54 \mathrm{~mm}$. y 0,36 mm. De ancho. (Aguirre, 2015).

Perisperma: Está constituido principalmente por granos de almidón y posee es de color amarillento y ocupa el $60 \%$ de la superficie de la semilla. (Mujica Sánchez\& Jacobsen, 2001)

Episperma: En ella se ubica la saponina que le da el sabor amargo al grano y cuya adherencia a la semilla es variable con los genotipos (Villacorta, 1976)

Embrión: Está formado por dos cotiledones y la radícula y constituye el 30\% del volumen total de la semilla, el cual envuelve al perisperma como un anillo, con una curvatura de $320^{\circ}$, es de color amarillo, cuya longitud puede alcanzarlos $3,54 \mathrm{~mm}$. y 0,36 mm. De ancho. (Aguirre, 2015).

Perisperma: Está constituido principalmente por granos de almidón y posee es de color amarillento y ocupa el $60 \%$ de la superficie de la semilla. (Mujica Sánchez\& Jacobsen, 2001)

También se conoce como el método seco, que consiste en someter el grano a un proceso de fricción para eliminar las capas periféricas del mismo (que son las que contienen las saponinas), en forma de polvo. Según (Bacigalupo, 1990) nos indican que en Perú y Bolivia se han hecho varias pruebas de desaponificado de quinua por este método. Sin embargo, cada uno de estos intentos presentó dificultades en los procesos seguidos, los que hicieron que los métodos no fueran los más eficientes. Uno de los estudios más interesantes dentro de este tema es la escarificadora diseñada y construida por Torres y Minaya, en 1980. En este caso, los granos de 


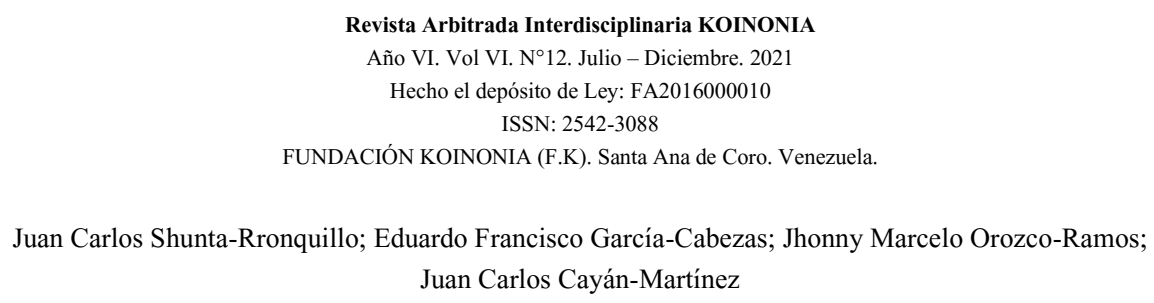

quinua son sometidos a un proceso combinado de efecto abrasivo y golpeado, con paletas giratorias sobre tamices estacionarios, los que recogen y separan el polvillo de saponina de los granos.

Con respecto, la máquina consta de tres cilindros dispuestos en tres bolillos, de tal forma que los granos en proceso pasan de un cilindro a otro por gravedad. Cada cilindro está provisto de 9 paletas escarificadoras y de 12 paletas transportadoras colocadas sobre el eje giratorio. Los granos que salen del último cilindro, reciben una corriente de aire, que ayuda a separar el polvo y afrechillo, antes de ser recogidos en la salida final. Según los autores, esta máquina escarificadora tiene una eficiencia del $95 \%$ y, los contenidos de saponina en el producto final oscilan entre el 0.04 y $0.25 \%$, dependiendo de la variedad utilizada como materia prima. (Torres, 1980)

\section{MATERIALES Y METODOS}

\section{Material de la máquina Escarificadora}

\section{Material del Eje}

Para el eje de la escarificadora se selecciona un acero AISI 4140, debido a que el eje no tendrá un contacto directo y prolongado con los granos de quinua, por lo que se procede a la selección de un material altamente resistente a los esfuerzos que este generará, además de ser menos costoso que un eje de acero inoxidable.

\section{Material de la estructura}

Debido a que la estructura no tiene un contacto directo sobre el procesamiento de la quinua se selecciona un acero ASTM A-36. 


\section{Selección del sistema de transmisión}

\section{Tabla 1.}

Resultado Sistema de Transmisión.

\begin{tabular}{|c|c|c|c|}
\hline \multirow{3}{*}{\begin{tabular}{l|} 
Selección \\
trasmisiones \\
por correa \\
trapecial.
\end{tabular}} & \multicolumn{3}{|l|}{ Descripción proceso } \\
\hline & \multicolumn{2}{|c|}{$\begin{array}{l}\text { Cálculo de la relación de } \\
\text { transmisión }\end{array}$} & \\
\hline & $\begin{array}{l}\text { Cálculo de la frecuencia de } \\
\text { trasmisión de la otra polea. }\end{array}$ & $\mathrm{n} 2=622$ & \\
\hline & \multirow{3}{*}{$\begin{array}{l}\text { Cálculo de la potencia de } \\
\text { diseño }\end{array}$} & Factor de servicio & 1 \\
\hline & & Potencia de diseño & $5,5 \mathrm{Hp}$ \\
\hline & & $\begin{array}{l}\text { Sección de correa } \\
\text { más adecuada. }\end{array}$ & $A$ \\
\hline & \multirow[t]{2}{*}{$\begin{array}{l}\text { Identificar la correa y las a } \\
\text { utilizar }\end{array}$} & \begin{tabular}{|lr} 
Cálculo de & la \\
relación & de \\
transmisión & del \\
apartado 1 & \\
\end{tabular} & $\mathbf{i}=2,75$ \\
\hline & & $\begin{array}{l}\text { Distancias entre } \\
\text { ejes en función del } \\
\text { tamaño de la } \\
\text { polea. }\end{array}$ & $C=600 \mathrm{~mm}$ \\
\hline & \multirow[t]{4}{*}{ Longitud de la correa } & \multirow[t]{3}{*}{$\mathrm{L}=1833 \mathrm{~mm}$} & $\mathrm{~N}^{\circ} 71$ \\
\hline & & & $\mathrm{Ln}=1811 \mathrm{~mm}$ \\
\hline & & & Perfil A \\
\hline & & \begin{tabular}{|lr} 
Distancias entre \\
centros de las \\
poleas (correas \\
normalizadas)
\end{tabular} & $\mathrm{Cc}=66 \mathrm{~mm}$ \\
\hline & $\begin{array}{l}\text { Determinación del arco de } \\
\text { contacto menor }\end{array}$ & $\begin{array}{ll}\text { Transmisión } & \text { con } \\
\text { correa abierta } & \end{array}$ & $\begin{array}{l}\propto 1 \\
=163,41 \\
>120^{\circ}\end{array}$ \\
\hline
\end{tabular}

Elaboración: Los autores. 


\section{Selección del rodamiento}

Se realizaron los cálculos pertinentes para la selección de los rodamientos, los cuales se detallas a continuación.

\section{Rodamiento A}

\section{Tabla 2.}

\section{Selección de Rodamiento A.}

\begin{tabular}{|l|l|}
\hline Reacción en eje $y$ & $290,5 \mathrm{~N}$ \\
\hline Reacción en eje z & $268,73 \mathrm{~N}$ \\
\hline Resultante & $303,8 \mathrm{~N}$ \\
\hline Carga estática & Co $=2,8 \mathrm{KN}$ \\
\hline Carga dinámica & C $=1,86 \mathrm{KN}$ \\
\hline Carga estática rodamiento J & Co $=\mathbf{1 9 K N}$ \\
\hline $\begin{array}{l}\text { Carga dinámica } \\
\text { rodamiento }\end{array}$ & C $=30,7 \mathrm{KN}$ \\
\hline fl de $\mathbf{6 , 4}$ & vida util de 38000 horas \\
\hline
\end{tabular}

Elaboración: Los autores.

\section{Rodamiento E}

\section{Tabla 3.}

\section{Selección de Rodamiento E.}

\begin{tabular}{|l|l|}
\hline Reacción en eje $y$ & $65,23 \mathrm{~N}$ \\
\hline Reacción en eje $z$ & $389,5 \mathrm{~N}$ \\
\hline Resultante & $400 \mathrm{~N}$ \\
\hline Carga estática & $\mathrm{Co}=\mathbf{3}, \mathbf{7 K N}$ \\
\hline Carga dinámica & $\mathrm{C}=\mathbf{2 , 4 6 \mathrm { KN }}$ \\
\hline Carga estática rodamiento & Co $=\mathbf{1 9 K N}$ \\
\hline Carga dinámica rodami ento & $\mathrm{C}=\mathbf{3 0}, \mathbf{7 K N}$ \\
\hline fl de $\mathbf{1 5}$ & vida util de 38000 horas \\
\hline
\end{tabular}

Elaboración: Los autores. 


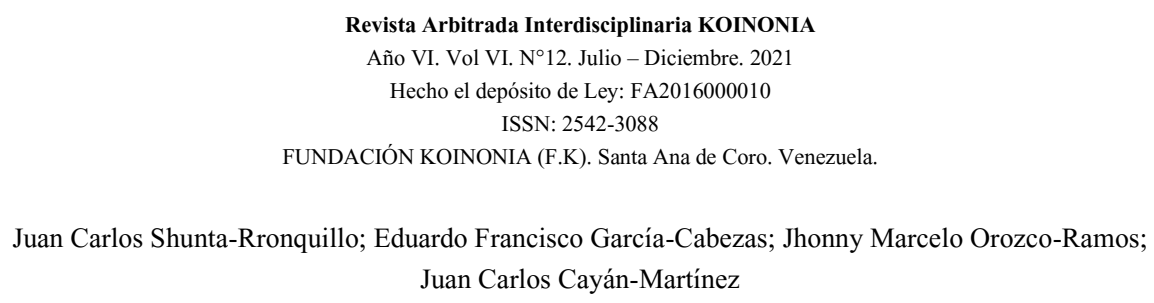

\section{Métodos Utilizados}

\section{Método mecánico por lavado o húmedo}

Consiste en someter al grano de quinua a un proceso de remojo y turbulencia, en agua circulante o fija en el recipiente de lavado, la saponina se elimina en el agua de lavado.

Consideraciones:

Tiempos prolongados de tratamiento con agua no mejoran el rendimiento de la extracción de saponina de quinua, por el contrario, incrementan la hidratación del grano, lo que dificulta o encarece el proceso de secado (Nieto \& Valdivia, 2001).

Si el contenido de humedad del producto desaponificado no excede de $27 \%$, el secado se puede hacer fácilmente con secadores solares o inclusive con exposición al ambiente (Nieto \& Valdivia, 2001).

\section{Método combinado}

Considerando los inconvenientes del desaponificado por el método húmedo y que el método seco no es eficiente para variedades de alto contenido de saponina, lo más aconsejado parecería ser la aplicación de un método combinado para la eliminación de saponificación de la quinua; es decir, primero se aplica un escarificado, con lo que se elimina un alto porcentaje de saponina y luego se somete a un lavado para eliminar el remanente. De esta forma, el grano no es expuesto excesivamente a la humedad y el proceso de secado es mucho más rápido y barato (Nieto \& Valdivia, 2001).

\section{Máquina escarificadora}

Es una máquina cuya función es pelar los granos de quinua, eliminando la saponina existente en la cascara del cereal, las máquinas existentes tienen mucha similitud debido a que se componen de una tolva que permite el ingreso del grano de quinua a la máquina, un sistema escarificador compuesta por un cilindro escarificador de paletas que se encarga de pelar el grano de quinua, un sistema de ventilación y separación de impurezas compuesta por un ventilador y una malla, por ultimo un ciclón que separa los granos del polvo, obteniendo así quinua escarificada. 
En la industria la mayoría de máquinas escarificadoras, entre sus principales elementos para la escarificación de la quinua se emplea el eje con paletas, está constituido por 6 u 10 barras de acero que presentan una forma exterior redondeada y cubierta de placas paralelas al eje, este tipo de eje escarificador "europeo", y sus características son: Diámetro D comprendido entre 400 y $600 \mathrm{~mm}$; la longitud variable entre 0.80 y $1.80 \mathrm{~m}$ y su velocidad generalmente regulable entre 400 y 1600 RPM.

\section{RESULTADOS}

\section{Máquina terminada.}

La máquina terminada consta con todos los elementos establecidos en el diseño de la maquinaria, satisfaciendo las exigencias para el procesamiento de la quinua.

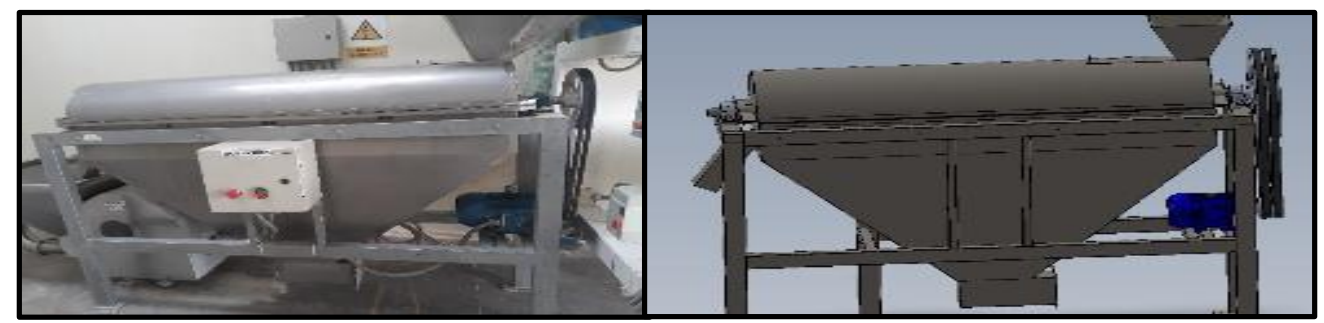

Figura 1. Máquina Terminada.

Fuente: Los autores.

\section{Prueba de funcionamiento}

Las pruebas de funcionamiento, fueron realizadas con una saca de Quinua de 120,2 libras.

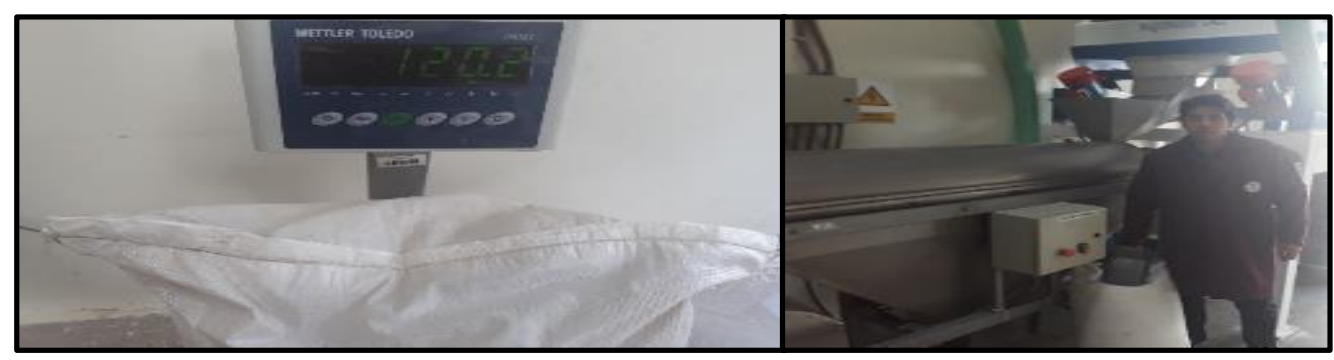

Figura 2. Pesaje de unidad de Prueba. Fuente: Los autores. 
Se procede el contenido de la unidad de prueba en la zaranda de quina para que esta sea la que alimente a la máquina escarificadora.

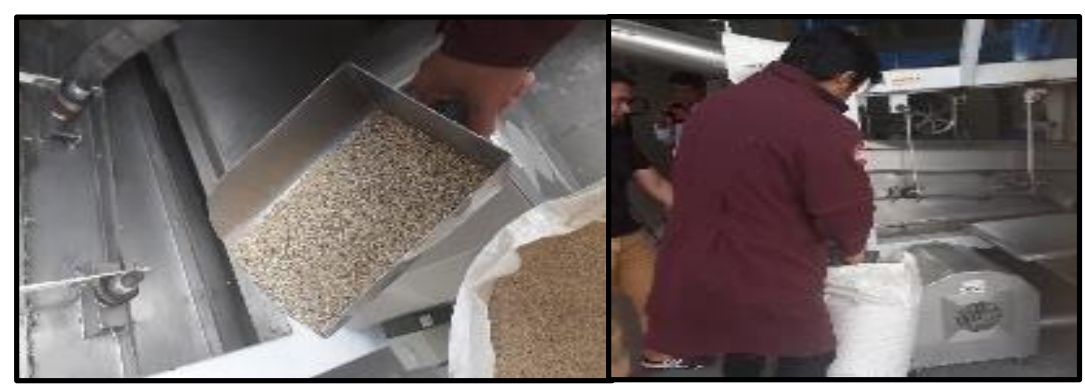

Figura 3. Alimentación a la zaranda dosificadora. Fuente: Los autores.

Se enciende la zaranda dosificadora y la máquina de quinua, para dar inicio a la escarificación de la quinua a continuación se muestran las imágenes del ingreso y salida de la Quinua.

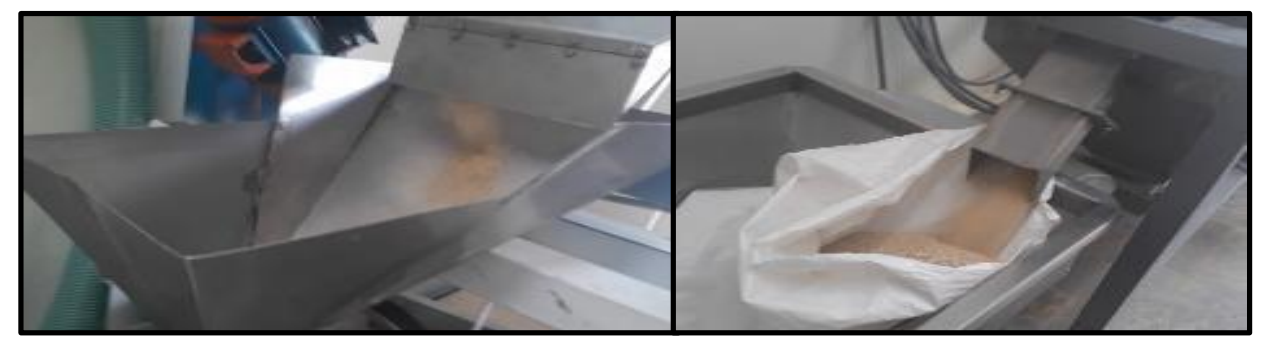

Figura 4. Ingreso y Salida de Saponina Fuente: Los autores. 
El proceso de escarificado tomo un tiempo de 15 minutos y 30 segundos, a continuación, se muestras las imágenes de la quinua y la saponina, luego de terminar el proceso. Se muestra el peso de 100, 2lb obtenido de quinua luego del escarificado. Pesaje de saponina, después del escarificado mediante la máquina.

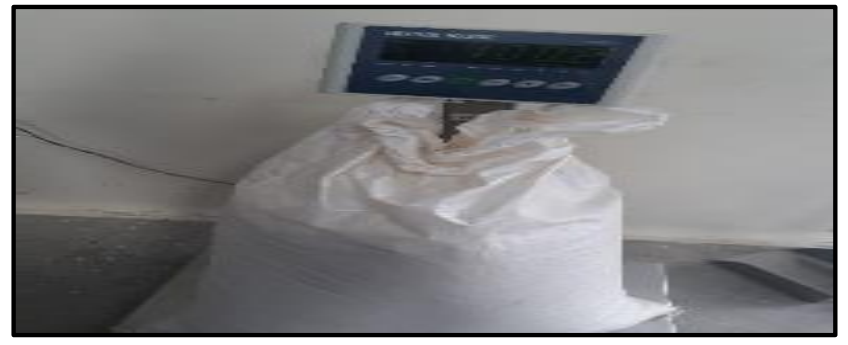

Figura 5. Quinua Escarificada. Fuente: Los autores.

Se muestra las imágenes del peso de 18,9lb. De saponina luego del proceso de escarificado.

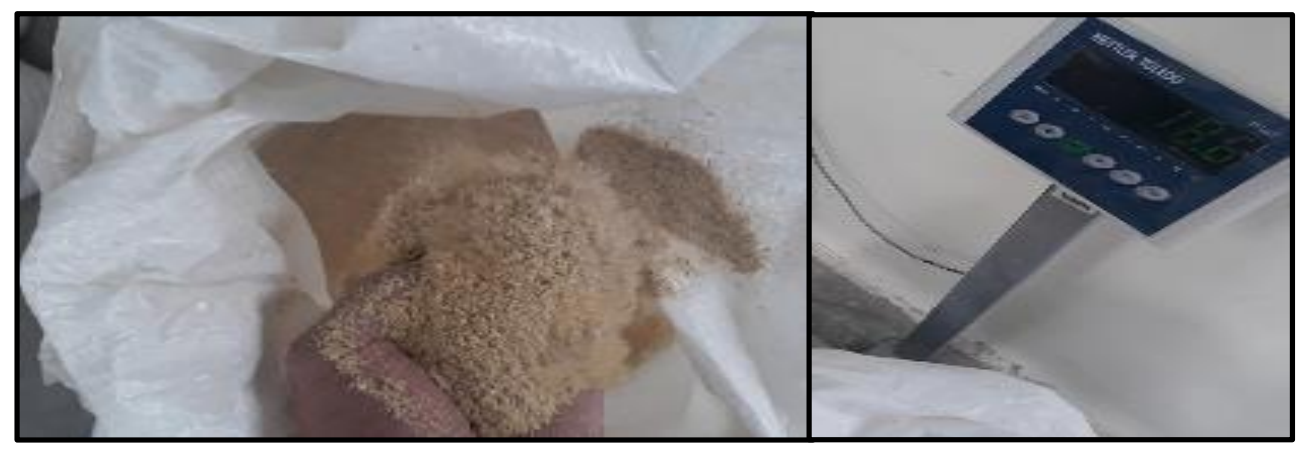

Figura 6. Residuo de Saponina. Fuente: Los autores. 


\section{Diagrama de Proceso General de la máquina escarificadora}

Tabla 4.

Diagrama de Proceso de la máquina escarificadora.

\begin{tabular}{|c|c|c|c|c|c|}
\hline \multicolumn{6}{|c|}{ DIAGRAMA DE PROCESO GENERAL } \\
\hline \multicolumn{2}{|c|}{ EMPPRESA } & ASOALIENU & \multirow{2}{*}{ \pm 1} & DEPARTAMENTO & PRODUCCIÓN \\
\hline \multicolumn{3}{|c|}{ Realizado por: Carlos Shunta } & & FECHA & $10 / 5 / 2017$ \\
\hline \multicolumn{3}{|c|}{ MAQUINARIA } & \multirow[b]{2}{*}{$\sqrt{5}$} & DIAGRAMA & PROCESO \\
\hline \multicolumn{3}{|c|}{ ESCARIFICADORA } & & MÉTODO & MECÁNICO \\
\hline $\mathrm{N}^{\circ}$ & \multicolumn{3}{|r|}{ DESCRIPCIÓN } & \multicolumn{2}{|c|}{ \# } \\
\hline \multicolumn{4}{|c|}{\begin{tabular}{l|l} 
1) Almacenamiento
\end{tabular}} & $10 \Rightarrow \square D$ & \\
\hline \multicolumn{4}{|c|}{2 Transporte desde la tolva hacia la escarificadora } & $O \Rightarrow \square D$ & 2 \\
\hline \multicolumn{4}{|c|}{\begin{tabular}{l|l|}
3 & Escarificado \\
\end{tabular}} & $\Rightarrow \square D$ & 930 \\
\hline \multicolumn{4}{|c|}{\begin{tabular}{l|l|}
11 & Transporte des de la escarificadora hacia la tolva de alm
\end{tabular}} & $O \Rightarrow \square D$ & 1 \\
\hline \multicolumn{4}{|c|}{\begin{tabular}{l|l|}
12 & Almacemaniento \\
\end{tabular}} & $20 \Rightarrow \square D$ & \\
\hline
\end{tabular}

Elaboración: Los autores.

\section{Tabla 5.}

Resultado máquina escarificadora.

\begin{tabular}{|c|c|c|}
\hline \multicolumn{3}{|c|}{ RESUMEN } \\
\hline SÍMBOLO & NÚMERO & TIEMPO(S) \\
\hline$\square$ & 2 & 3 \\
\hline 0 & 1 & 930 \\
\hline$D$ & 0 & 0 \\
\hline$\nabla$ & 2 & 0 \\
\hline$\square$ & 0 & 0 \\
\hline & & 933 \\
\cline { 3 - 3 } & &
\end{tabular}

Elaboración: Los autores. 


\section{Comparación método rudimentario vs máquina escarificadora}

Tabla 6.

Resumen del diagrama de proceso, método rudimentario.

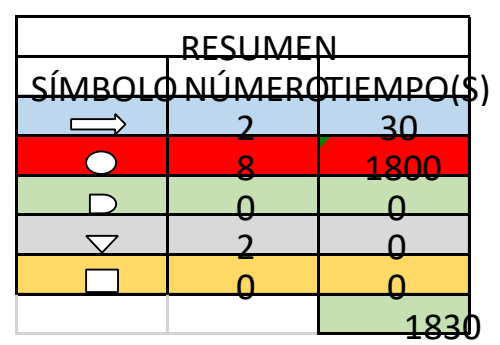

Elaboración: Los autores.

\section{Tabla 7.}

Resumen del diagrama de proceso, método maquina escarificadora.

\begin{tabular}{|c|c|c|}
\hline \multicolumn{3}{|c|}{ RESUMEN } \\
\hline SÍMBOLO & NÚMERO & TIEMPO(S) \\
\hline$\square$ & 2 & 3 \\
\hline 0 & 1 & 930 \\
\hline$D$ & 0 & 0 \\
\hline$\nabla$ & 2 & 0 \\
\hline$\square$ & 0 & 0 \\
\hline & & 933 \\
\cline { 3 - 3 } & &
\end{tabular}

Elaboración: Los autores.

\section{CONCLUSIONES}

Mediante el análisis de los cuadros de resumen de los diagramas de proceso, método rudimentario y método actual, se puede apreciar que mediante la implementación de la máquina escarificadora el proceso de escarificado en la máquina es de una operación, mientras que en el método rudimentario es de ocho operaciones.

Con la reducción de actividades se demuestra la optimización del proceso de escarificado, es afirmativa, debido a que no hay tiempos de espera, ni tiempos muertos en el proceso de escarificado, en comparación del método rudimentario, 


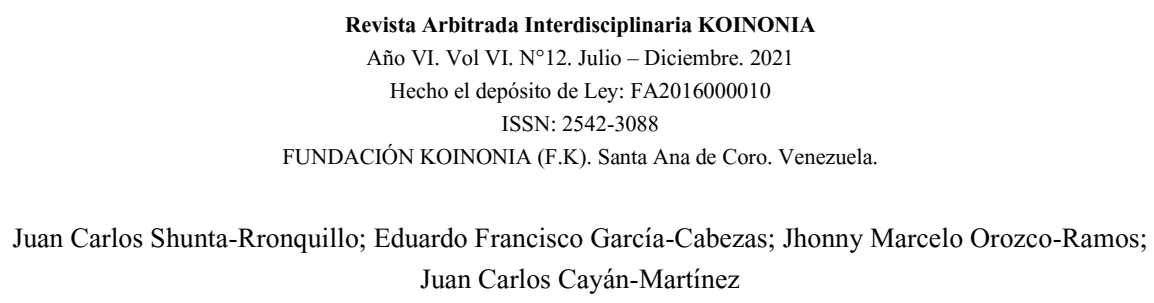

existes demoras en el proceso, debido a que para poder separar la saponina del grano luego de haber friccionado los mismos se necesita de una corriente de viento que ayude a la separación, media que realizan los agricultores en las horas de la tarde para aprovechar las corrientes de aire generadas.

Otro punto relevante para tomar en cuenta en la optimización del proceso de escarificado de la Quinua es el volumen de producción que se genera, para determinar la mejora se realiza pruebas de escarificado manual y mecánico a una muestra de $120 \mathrm{lb}$. Obteniendo los resultados que nos expresan los cuadros de resumen. método manual nos arroja un tiempo de 30 minutos y 30 segundos, la máquina escarificadora un tiempo de 15 minutos 30 segundos, equivalente a la reducción de un $49,18 \%$ en el proceso de escarificado.

\section{FINANCIAMIENTO}

Monetario.

\section{AGRADECIMIENTO}

A los colaboradores y a los trabajadores de la Empresa ASOALIENU, que prestaron su apoyo para la realización de esta investigación adscrita Escuela Superior Politécnica de Chimborazo Facultad de Mecánica de la Escuela de Ingeniería Industrial. Riobamba - Ecuador.

\section{REFERENCIAS CONSULTADAS}

Aguirre, M. (2015). Efectos de la temperatura sobre las características físicas de granos actuales de Chenopodium Quinoa Willd (Quínoa). [Effects of temperature on the physical characteristics of current grains of Chenopodium Quinoa Willd (Quinoa)]. Editor: Universidad Autónoma de Madrid. Departamento de Prehistoria y Arqueología. Revista BAEX. Boletín de Arqueología Experimental. Número 10. 2015, p.13-26. 


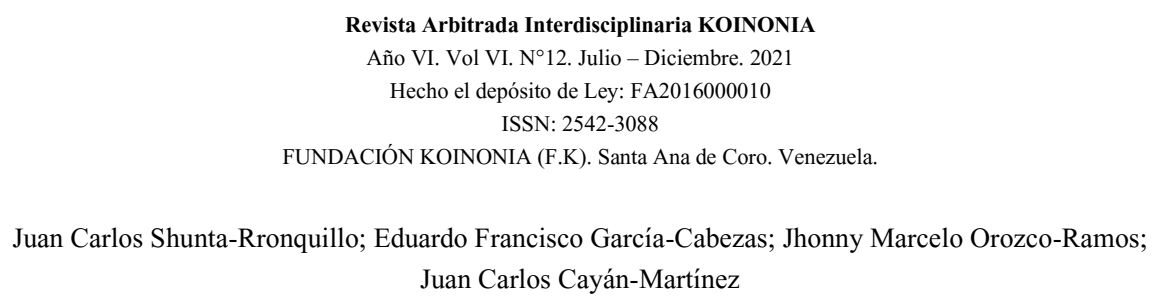

Alarcón, Á. (2014). Reconstrucción de una trilladora agrícola con adaptación de un motor de combustión interna para una microempresa familiar en el cantón Alausí, [Reconstruction of an agricultural thresher with adaptation of an internal combustion engine for a family micro-business in Alausí canton]. Quito: UIDE. Quito. Ecuador.

Candia, I. \&. Danz, I. (2016). Diseño y evaluación de una escarificadora para la extracción de saponina de la Quinua-Región Puno. [Design and evaluation of a scarifier for the extraction of saponin from the Quinoa-Puno Region ]. S.L.:S.N. Puno. Perú.

Mujica, S \& Jacobsen,H. (2006). La quinua (Chenopodium quinoa Willd.) y sus parientes silvestres. [Quinoa (Chenopodium quinoa Willd.) And its wild relatives]. Botánica Económica de los Andes Centrales Editores: M. Moraes R., B. Øllgaard, L. P. Kvist, F. Borchsenius \& H. Balslev Universidad Mayor de San Andrés, La Paz, 2006: 449-457. Bolivia.

Nieto, C. \& Vimos, C. (1994). Producción y procesamiento de Quinua en Ecuador. [Production and processing of Quinoa in Ecuador]. Programa de cultivos Andinos, Estación Experimental Santa Catalina, INIAP. Proyecto de cooperación técnica 3P-90-0160. Quito, Ecuador.

Carrasco, R. \&. Zelenda. C. (2008). Determinación de la capacidad antioxidante y compuestos fenólicos de cereales andinos: quinua (Chenopodium quinoa), kañiwa (Chenopodium pallidicaule) y kiwicha (Amaranthus caudatus). S.L.: Revista de la Sociedad Química del Perú. Lima, Perú.

Orozco, D. (2014). Situación macroeconómica de la quinua en el Ecuador y su capacidad exportable período 2007-2011. [Macroeconomic situation of quinoa in Ecuador and its exportable capacity 2007-2011 period]. Quito: s.n. Quito, Ecuador.

Ramos, A. (2006). Diseño de una máquina escarificadora de quinua para mejorar la producción de quinua en las comunidades campesinas de Sicaya. [Design of a quinoa scarifying machine to improve quinoa production in rural communities of Sicaya]. Tesis Pregrado ed. Huancayo: S.N. SICAYA. Perú.

Torres, H. \&. Minaya. I. (1980). Escarificadora de Quinua. [Quinoa Scarifier]. Editor Instituto Interamericana de Ciencias Agrícolas. S.L.: Publicaciones misceláneas. Lima. Perú. 
Revista Arbitrada Interdisciplinaria KOINONIA

Año VI. Vol VI. N${ }^{\circ}$ 12. Julio - Diciembre. 2021

Hecho el depósito de Ley: FA2016000010 ISSN: 2542-3088

FUNDACIÓN KOINONIA (F.K). Santa Ana de Coro. Venezuela.

Juan Carlos Shunta-Rronquillo; Eduardo Francisco García-Cabezas; Jhonny Marcelo Orozco-Ramos;

Juan Carlos Cayán-Martínez

Villacorta, L. \&. Rodríguez. V. (1976). Anatomía del grano de Quinua (Chenopodium quinoa Wild.). [Quinoa grain anatomy]. Editor Universidad Nacional del Altiplano. Puno (UNAP). Puno. Perú.

(C2020 por el autor. Este artículo es de acceso abierto y distribuido según los términos y condiciones de la licencia Creative Commons Atribución-NoComercial-Compartirlgual 4.0 Internacional (CC BY-NC-SA 4.0) (https://creativecommons.org/licenses/by-nc-sa/4.0/). 